\title{
Resistance profile of drugs composing the "shorter" regimen for multidrug-resistant tuberculosis in Brazil, 2000-2015
}

To the Editor:

The difficulties in managing multidrug-resistant tuberculosis (MDR-TB) and extensively drug-resistant (XDR) TB are well known. The regimens are very expensive, often toxic, and require up to 24 months to achieve an acceptable probability of success [1-3].

Based on successful experiences in different settings, the World Health Organization (WHO) recently provided recommendations on the prescription of a new "shorter" regimen to treat MDR-TB, which is also known as the "Bangladesh regimen" [4-8]. The regimen is composed of kanamycin, moxifloxacin (gatifloxacin being the fluoroquinolone originally used in the "Bangladesh" regimen), prothionamide, clofazimine, pyrazinamide, high-dose isoniazid, and ethambutol for an initial period (4-6 months), then moxifloxacin, clofazimine, pyrazinamide and ethambutol for the continuation phase (5 months) [8].

The regimen has interesting characteristics. It is considerably cheaper than the traditional "long" regimen (<USD1000 versus >USD20000 or more), has a much shorter duration (9-12 months), and achieves relapse-free success rates exceeding $90 \%$ (the positive outcomes of the traditional "longer" regimen are on the order of $50-60 \%)$ [8].

The regimen is indicated for patients not previously treated with second-line drugs and unlikely to be resistant to them. WHO recommended the extensive use of rapid molecular testing for the detection of resistances to second-line drugs (Geno Type MTBDRsl) to allow prescription of this regimen to the patients who can benefit from it without risk of developing further drug resistance [8].

Recently, several contributions tried to estimate the proportion of eligible patients in different geographical settings. This ranged from $\sim 10 \%$ in Europe to $50 \%$ in Pakistan [9-12].

No countrywide information in this sense is available from Latin America. As part of an ongoing collaboration project between the European Respiratory Society (ERS), the Brazilian Society of Respiratory Medicine (SBPT) and the Brazilian National TB Programme, we analysed the information provided by the Brazilian national register (SITE TB), including all patients needing a treatment different from the standard 6 month regimen for (drug-susceptible) TB cases.

The aim of this study is to describe the resistance profile of the drugs composing the "shorter" regimen among MDR-TB cases diagnosed between 2000 and 2015.

In Brazil, the MDR-TB regimen is standardised in the majority of cases [13]. Originally, it contained clofazimine and ofloxacin, but in 2006 pyrazinamide replaced clofazimine and in 2010 levofloxacin replaced ofloxacin as the backbone fluoroquinolone (5 months with amikacin or streptomycin (five times a week for 1 month and three times a week for the following 4 months), ethambutol, levofloxacin, pyrazinamide and terizidone, and 12 months with ethambutol, levofloxacin and terizidone).

On this basis we analysed, separately, individuals enrolled between 2000 and 2010 from those treated between 2011 and 2015.

Absolute frequencies (percentages) and medians (interquartile ranges; IQRs) were used to describe qualitative and quantitative variables. Point and interval (95\% CI) estimates were adopted to assess the drug resistance prevalence. Chi-squared or Fisher exact and Mann-Whitney tests were carried out to

@ERSpublications

The first nationwide report on the drug resistance profile of the drugs composing the WHO "shorter" regimen in Brazil http://ow.ly/XfJa3096FsT

Cite this article as: Dalcolmo M, Gayoso R, Sotgiu G, et al. Resistance profile of drugs composing the "shorter" regimen for multidrug-resistant tuberculosis in Brazil, 2000-2015. Eur Respir J 2017; 49: 1602309 [https://doi.org/10.1183/13993003.02309-2016]. 
assess statistically significant differences for qualitative and quantitative variables, when appropriate. A p-value of less than 0.05 was considered statistically significant. All statistical analyses were performed using STATA version 14 (StataCorp LP 2015; College Station, TX, USA).

Of 6833 patients enrolled, 4477 (66\%) were males, with a median (IQR) age of 39 (29-49) years.

The HIV/TB co-infected patients amounted to 7.4\% (240/3251) in the period 2000-2010 and 11.7\% (369/ 3156) between 2011 and 2015 ( $\mathrm{p}<0.001$ ). No statistically significant differences were detected between the above-mentioned subgroups in terms of sex. Patients treated between 2011 and 2015 were significantly older (median (IQR) age: 38 (29-48) versus 39 (29-50) years $(\mathrm{p}=0.03)$.

Drug susceptibility testing (DST) was performed using solid media (Lowenstein-Jensen, Middlebrook 7H10/11) until 2010, and then liquid media (Mycobacterial Growth Indicator Tube (MGIT ${ }^{\mathrm{TM}}$ ); BD Diagnostic, Sparks, MD, USA) in quality-assured laboratories.

In Brazil, DST is usually carried out for pyrazinamide and ethambutol. In the period 2000-2010, ethionamide was tested frequently, while in the period 2011-2015 the number of patients tested for this drug was lower, as well as the number of those tested for pyrazinamide. In the first period, fluoroquinolones and injectables were tested in a few patients (100 and 115, respectively), but in the period 2011-2015 DST was performed for these drugs more frequently. DST was not performed for clofazimine.

Overall, a high prevalence of drug resistance was found for pyrazinamide (1474/2950; 50.0\%, 95\% CI $48.2-51.8 \%)$, followed by fluoroquinolones (369/1072; 34.4\%, 95\% CI 31.6-37.3\%), ethambutol (2111/6408; 32.9\%, 95\% CI 31.8-34.1\%), ethionamide (488/1690; 28.9\%, 95\% CI 26.7-31.0\%), kanamycin (112/941; $11.9 \%$, 95\% CI 9.8-14.0\%) and amikacin (134/1039; 12.9\%, 95\% CI 10.9-14.9\%) (table 1).

Comparing the periods 2000-2010 and 2011-2015, the prevalence of drug resistance decreased for all drugs, with a significant decline for pyrazinamide $(51.5 \%$ versus $46.7 \%$; $\mathrm{p}=0.01)$, ethambutol $(37.9 \%$ versus $28.3 \%$; $\mathrm{p}<0.0001)$ and ethionamide $(29.7 \%$ versus $19.1 \%$; $\mathrm{p}=0.01)$. The prevalence of resistance to fluoroquinolones increased from $30 \%$ to $34.9 \%$, without any statistical significance (probably due to increased DST frequency).

The individual DST pattern was somehow heterogeneous during the entire study period (table 1).

Focusing on the drugs composing the "shorter" regimen, in the period 2000-2010, of the 1319 MDR-TB patients undergoing DST for pyrazinamide, ethambutol and ethionamide, 150 (11.4\%) were resistant to three drugs. Although the proportion of cases tested was low (table 1), resistance to fluoroquinolones was $30 \%$ (largely reflecting the DST for ofloxacin, which was performed on 28 of 100 strains tested for fluoroquinolones), $22.2 \%$ for kanamycin and $15.9 \%$ for amikacin.

In the period 2011-2015, the prevalence of resistance was $46.7 \%$ for pyrazinamide, $28.3 \%$ for ethambutol, $34.9 \%$ for fluoroquinolones (339/972, again largely reflecting the DST for ofloxacin), $12.6 \%$ for amikacin (120/951) and $11.6 \%$ for kanamycin (106/914).

The study has several limitations, because it is based on programmatic information in a setting where it is not possible to perform a complete DST for each patient. Furthermore, although performed in quality-controlled laboratories belonging to the WHO network, the reliability of DST for some of the drugs tested (pyrazinamide and ethionamide, among others) requires some caution in evaluating the data. Finally, we cannot exclude a selection bias related to the limited DST coverage for some of the drugs.

TABLE 1 Nationwide resistance to the drugs composing the World Health Organization "shorter" regimen in Brazil, 2000-2015

\begin{tabular}{|c|c|c|c|c|c|c|c|}
\hline \multirow[t]{2}{*}{ Drug tested } & \multicolumn{2}{|c|}{$2000-2010$} & \multicolumn{2}{|c|}{ 2011-2015 } & \multirow[t]{2}{*}{ p-value } & \multicolumn{2}{|c|}{ Overall 2000-2015 } \\
\hline & $\begin{array}{c}\text { Resistant/ } \\
\text { tested } \mathrm{n}\end{array}$ & $\begin{array}{l}\text { Resistant } \\
\%(95 \% \mathrm{Cl})\end{array}$ & $\begin{array}{l}\text { Resistant/ } \\
\text { tested } \mathrm{n}\end{array}$ & $\begin{array}{c}\text { Resistant \% } \\
(95 \% \mathrm{CI})\end{array}$ & & $\begin{array}{c}\text { Resistant/ } \\
\text { tested } \mathrm{n}\end{array}$ & $\begin{array}{c}\text { Resistant \% } \\
(95 \% \mathrm{CI})\end{array}$ \\
\hline Ethambutol & $1177 / 3103$ & $37.9(36.2-39.6)$ & $934 / 3305$ & $28.3(26.8-29.8)$ & $<0.0001$ & $2111 / 6408$ & $32.9(31.8-34.1)$ \\
\hline Fluoroquinolones" & $30 / 100$ & $30.0(21.0-39.0)$ & $339 / 972$ & $34.9(31.9-37.9)$ & 0.33 & $369 / 1072$ & $34.4(31.6-37.3)$ \\
\hline Levofloxacin & $2 / 7$ & $28.6(-4.9-62.1)$ & $3 / 19$ & $15.8(-0.6-32.2)$ & 0.59 & $5 / 26$ & $19.2(4.1-34.3)$ \\
\hline Amikacin & $14 / 88$ & $15.9(8.3-23.5)$ & $120 / 951$ & $12.6(10.5-14.7)$ & 0.38 & $134 / 1039$ & $12.9(10.9-14.9)$ \\
\hline Kanamycin & $6 / 27$ & $22.2(6.5-37.9)$ & $106 / 914$ & $11.6(9.5-13.7)$ & 0.09 & $112 / 941$ & $11.9(9.8-14.0)$ \\
\hline Ethionamide & $464 / 1564$ & $29.7(27.4-32.0)$ & $24 / 126$ & $19.1(12.2-26.0)$ & 0.01 & $488 / 1690$ & $28.926 .7-31.01$ \\
\hline
\end{tabular}

\#: resistance to any fluoroquinolone. 
However, the database is quality-controlled and contains a large number of comprehensive nationwide records (over 6000 cases) from a high-TB-burden country. To our knowledge, this is the first nationwide report on the drug resistance profile of the drugs composing the WHO "shorter" regimen in a high-burden country.

The study results allow us to draw some important conclusions.

First, that the programmatic approach to MDR-TB through standardised regimens seems not to have contributed to increasing the prevalence of resistance to the drugs used [13].

Secondly, although the data available in Brazil do not allow us to exactly predict the proportion of patients who are eligible for the shorter regimen, it is very likely to be higher than that recently reported in Europe (10\%) and Pakistan (50\%) [9-12]. Considering that i) about 30\% of the cases tested for fluoroquinolones are resistant to it, ii) recently, about $12 \%$ of patients were found to be resistant to second-line injectables (although not all Mycobacterium tuberculosis strains were tested for fluoroquinolones and second-line injectables), and iii) there is apparently no increase in resistance trend, we might optimistically assume that up to $50-55 \%$ of the MDR-TB population in Brazil is likely to be susceptible to both fluoroquinolones and injectables and, therefore, potentially suitable for the "shorter" regimen. It is, therefore, mandatory that susceptibility to the drugs composing the regimen is confirmed before prescribing it.

A recent study demonstrated that the "shorter" regimen might reduce, in 8 years, the MDR-TB incidence by between $-23 \%$ and $-2 \%$ based on several assumptions, including the long-term efficacy of the "shorter" regimen, the degree of scale-up of MDR-TB treatment, and the likely transmission of $M$. tuberculosis drug-resistant strains within the community $[14,15]$. The results of the present study contribute to complement the available evidence.

Thirdly, in agreement with the WHO recommendations [8], it is important that national TB programmes in countries such as Brazil expand their capacity for rapid molecular testing (MTBDRsl) and DST for second-line drugs (at least fluoroquinolones and injectables) to enable clinicians to prescribe the "shorter" regimen to the right patients.

Margareth Dalcolmo ${ }^{1,8}$, Regina Gayoso ${ }^{1,8}$, Giovanni Sotgiu $\oplus^{2,8}$, Lia D’Ambrosio ${ }^{3,4,8}$, Jorge Luiz Rocha ${ }^{1}$, Liamar Borga ${ }^{1}$, Fatima Fandinho ${ }^{5}$, Jose Ueleres Braga ${ }^{6}$, Denise Arakaki Sanchez, Fernanda Dockhorn ${ }^{7}$, Rosella Centis ${ }^{3}$ and Giovanni Battista Migliori $\varpi^{3}$

${ }^{1}$ Hélio Fraga Reference Center, Fiocruz/MoH, Rio de Janeiro, Brazil. ${ }^{2}$ Clinical Epidemiology and Medical Statistics Unit, Department of Biomedical Sciences, University of Sassari - Research, Medical Education and Professional Development Unit, AOU Sassari, Sassari, Italy. ${ }^{3}$ World Health Organization Collaborating Centre for TB and Lung Diseases, Maugeri Institute, IRCCS Tradate, Tradate, Italy. ${ }^{4}$ Public Health Consulting Group, Lugano, Switzerland. ${ }^{5}$ National Mycobacteriology Laboratory, CRPHF, National TB Reference Center, Fiocruz/MoH, Rio de Janeiro, Brazil. ${ }^{6}$ National Public Health School, Epidemiology Department, Fiocruz/MoH, Rio de Janeiro, Brazil. ${ }^{7}$ National Tuberculosis Control Programme, SVS, Ministry of Health, Brazil. ${ }^{8}$ These authors contributed equally to this work.

Correspondence: Giovanni Battista Migliori, World Health Organization Collaborating Centre for Tuberculosis and Lung Diseases, Maugeri Institute, IRCCS Tradate, Via Roncaccio 16, Tradate, Varese 21049, Italy.

E-mail: giovannibattista.migliori@icsmaugeri.it

Received: Nov 242016 | Accepted after revision: Jan 092017

Conflict of interest: None declared.

\section{References}

1 Diel R, Vandeputte J, de Vries G, et al. Costs of tuberculosis disease in the European Union: a systematic analysis and cost calculation. Eur Respir J 2014; 43: 554-565.

2 Migliori GB, Sotgiu G, Gandhi NR, et al. Drug resistance beyond extensively drug-resistant tuberculosis: individual patient data meta-analysis. Eur Respir J 2013; 42: 169-179.

3 Falzon D, Gandhi N, Migliori GB, et al. Resistance to fluoroquinolones and second-line injectable drugs: impact on multidrug-resistant TB outcomes. Eur Respir J 2013; 42: 156-168.

4 Aung KJ, Van Deun A, Declercq E, et al. Successful '9-month Bangladesh regimen' for multidrug-resistant tuberculosis among over 500 consecutive patients. Int J Tuberc Lung Dis 2014; 18: 1180-1187.

5 Kuaban C, Noeske J, Rieder HL, et al. High effectiveness of a 12-month regimen for MDR-TB patients in Cameroon. Int J Tuberc Lung Dis 2015; 19: 517-524.

6 Piubello A, Harouna SH, Souleymane MB, et al. High cure rate with standardised short-course multidrug-resistant tuberculosis treatment in Niger: no relapses. Int J Tuberc Lung Dis 2014; 18: 1188-1194.

7 Van Deun A, Maug AK, Salim MA, et al. Short, highly effective, and inexpensive standardized treatment of multidrug-resistant tuberculosis. Am J Respir Crit Care Med 2010; 182: 684-692.

8 World Health Organization. WHO Treatment Guidelines for Drug-Resistant Tuberculosis. 2016 Update. WHO/HTM/TB/2016.04. Geneva, World Health Organization, 2016.

9 Sotgiu G, Tiberi S, D'Ambrosio L, et al. Faster for less, the new 'shorter' regimen for multidrug-resistant tuberculosis. Eur Respir J 2016; 48: 1503-1507.

10 van der Werf MJ, Hollo H, Ködmön C, et al. Eligibility for shorter treatment of multidrug-resistant tuberculosis in the European Union. Eur Respir J 2017; 49: 1601992. 
11 Sotgiu G, Tiberi S, D’Ambrosio L, et al. WHO recommendations on shorter treatment of multidrug-resistant tuberculosis. Lancet 2016; 387: 2486-2487.

12 Javaid A, Ahmad N, Khan AH, et al. Applicability of World Health Organization's recommended new shorter regimen in a multidrug-resistant tuberculosis high burden country. Eur Respir J 2017; 49: 1601967.

13 Dalcolmo MP, Fortes A, Melo FF, et al. Estudo de efetividade de esquemas alternativos para o tratamento da tuberculose multirresistente no Brasil. Jornal de Pneumologia 1999; 25: 70-77.

14 Kendall EA, Fojo AT, Dowdy DW. Expected impact of adopting a 9-month regimen for multidrug-resistant tuberculosis: a population modelling analysis. Lancet Respir Med 2017; 5: 191-199.

15 Sotgiu G, Migliori GB. Effect of the short-course regimen on the global epidemic of multidrug-resistant tuberculosis. Lancet Respir Med 2017; 5: 159-161. 